\title{
Preoperative evaluation of renal artery anatomy using computed tomography angiography to guide the superselective clamping of renal arterial branches during a laparoscopic partial nephrectomy
}

\author{
XIANGJUN MENG ${ }^{1,2}$, QIWU MI ${ }^{2}$, SHAOWEI FANG ${ }^{2}$ and WEIDE ZHONG ${ }^{1}$ \\ ${ }^{1}$ Southern Medical University, Guangzhou, Guangdong 510515; ${ }^{2}$ Department of Urology, Dongguan People's Hospital, \\ Dongguan, Guangdong 523059, P.R. China
}

Received August 1, 2014; Accepted April 24, 2015

DOI: $10.3892 / \mathrm{etm} .2015 .2500$

\begin{abstract}
The aim of the present study was to evaluate the clinical value of preoperative computed tomography angiography (CTA) imaging for guiding the superselective clamping of renal arterial branches during a laparoscopic partial nephrectomy (LPN). A total of 42 patients with renal masses of $<4 \mathrm{~cm}$, who had undergone a LNP, were retrospectively enrolled in the study between May 2008 and December 2013. CTA was performed preoperatively and the renal arterial anatomy was evaluated independently by two radiologists. Surgical observations, including the number, location and branching patterns of the renal arteries, were documented by the surgeon. Subsequently, the description of the renal arterial anatomy obtained using CTA was compared with the actual renal vascular structure observed during surgery. In total, 42 patients successfully underwent an LNP with superselective clamping of the renal arterial branches. The accuracy of CTA for the detection of the renal artery and renal tumor-feeding branches was 97.6 and $85.7 \%$, respectively. The CTA images facilitated the correct identification of 36/36 single renal arteries (100\%), while 5/6 renal accessory arteries were preoperatively detected using the CTA images. Statistical analyses indicated no statistically significant differences between the preoperative CTA and surgical LPN results for the number of clamped segmental arteries. Therefore, CTA was demonstrated to accurately visualize renal vascular anatomy and provide an advantage by enhancing the detection of tumor-feeding arteries. Thus, the use of CTA may facilitate improved segmental renal artery clamping during LPN.
\end{abstract}

Correspondence to: Professor Weide Zhong, Southern Medical University, 1,838 Guangzhou North Avenue, Guangzhou, Guangdong 510515, P.R. China

E-mail: dongguan0508@foxmail.com

Key words: angiography, laparoscopy, partial nephrectomy, segmental renal artery, vasculature

\section{Introduction}

Partial nephrectomy is currently the standard treatment for low-stage renal cell carcinoma, providing oncological outcomes that are comparable to radical nephrectomy (1). Recently, advances in surgical techniques have resulted in the development of minimally invasive surgical approaches (2). Application of a laparoscopic partial nephrectomy (LPN) facilitates a reduced hospital duration, and decreased operative bleeding and operative duration when compared with an open partial nephrectomy procedure (3). However, a LPN remains a challenging procedure for surgeons to perform.

In general, the main renal artery is clamped during a LPN in order to reduce hemorrhage. Clamping of the main renal artery allows for improved visualization, which enables the repair of the renal collecting system and the resection of the tumor (4). However, hilar occlusion may result in renal ischemic injury and impair renal function. Loss of renal function occurs if the duration of warm ischemia is $>28 \mathrm{~min}$ (5). A number of techniques have been developed with the aim of minimizing warm ischemic injury $(2,6,7)$. The technique of renal segmental artery clamping involves the selective clamping of renal arterial branches, and may eliminate renal ischemia from the entire kidney and minimize renal functional loss (4). It is crucial that surgeons obtain preoperative knowledge of the renal arterial anatomy in order to successfully perform selective renal arterial branch clamping.

Computed tomography angiography (CTA) has previously been used to accurately visualize renal vascular anatomy $(8,9)$. In the present study, patients underwent a LPN with CTA to enable the superselective clamping of the renal arterial branches. The aim of the present study was to evaluate the clinical value of CTA in the preoperative assessment of renal arterial anatomy by comparing the preoperative CTA results with the surgical observations obtained during LPN.

\section{Patients and methods}

Ethical approval and patient selection. The study protocol was approved by the Dongguan People's Hospital ethical committee (Dongguan, China), and patients provided written 
informed consent for the publication of the present case details.

A total of 42 patients with renal masses of $<4 \mathrm{~cm}$ were retrospectively enrolled in the study between May 2008 and December 2013. All the patients had undergone a LPN with superselective clamping of the renal arterial branches. The inclusion criteria for the LPN was a tumor size of $<4 \mathrm{~cm}$. Patients were excluded from the study if the LPN surgical observations were insufficiently documented to permit comparison with the preoperative CTA images.

CTA protocol. Patients underwent a preoperative CTA examination. A Brilliance iCT multidetector spiral CT scanner (Philips Medical Systems, Inc., Cleveland, OH, USA) was used to examine the 42 patients. CTA was performed using the following parameters: Standard modality; gantry rotation, $0.4 \mathrm{sec}$; pitch, 0.915; tube potential, $120 \mathrm{kV}$; tube current, $300 \mathrm{~mA}$; collimation, 128x0.625 mm; matrix, 512x512; thickness, $0.9 \mathrm{~mm}$; increment, $-0.45 \mathrm{~mm}$; and dose length product, $802.3 \mathrm{mGy}^{\bullet} \mathrm{cm}$.

An Ultravist contrast agent (iopromide, $370 \mathrm{mgI} / \mathrm{ml}$; Bayer HealthCare Pharmaceuticals, Berlin, Germany) was administered via a CT high-pressure syringe, with a moderate injection rate $(4-5 \mathrm{ml} / \mathrm{sec})$ and dose $(1.0-1.5 \mathrm{ml} / \mathrm{kg})$. Following an unenhanced CT examination of the abdomen, a high-resolution contrast-enhanced scan was performed on the kidneys in the arterial, venous and urographic phases.

Images were postprocessed using an Extended Brilliance Workspace independent workstation (version 4.5; Philips Medical Systems, Inc.) to obtain multiplanar reconstructions and maximum intensity projections, in addition to surface and volume renderings.

Image analysis. CTA images were independently reviewed by two radiologists that were blinded to the study. The number, location and branching patterns of the renal arteries were documented and the extrarenal length of the tumor-feeding arteries (from the first branch of the main renal artery to the distal part of segmental artery reaching the renal capsule) was measured. For supernumerary renal arteries, the artery with the maximum diameter was recorded as the main renal artery and all others were classified as renal accessory arteries.

Surgical protocol. All 42 cases underwent a LPN via the retroperitoneal approach. Surgery was performed following the CTA examination by a surgeon with 10 years experience of performing LPNs. Tumor-feeding arteries were preoperatively selected by two radiologists on the basis of the CTA images. Following the superselective renal arterial clamping of the tumor-feeding arteries, the tumors were resected. For each patient the surgeon recorded a number of surgical observations, including the number, location, extrarenal length and branching patterns of the renal arteries. In addition, clinical, perioperative and follow-up outcomes (9-75 months) were recorded.

Statistical analysis. LPN surgical observations were considered to be the reference standard for comparison. Statistical analysis was performed using SPSS software, version 17.0
Table I. Patient demographics, tumor characteristics, surgical details and outcome.

\begin{tabular}{|c|c|c|}
\hline Variables & Mean \pm SD & Median (range) \\
\hline Patients (n) & 42 & - \\
\hline Age (years) & $58.4 \pm 14.8$ & $56(45-72)$ \\
\hline Male/female (n) & $24 / 18$ & - \\
\hline $\operatorname{BMI}\left(\mathrm{kg} / \mathrm{m}^{2}\right)$ & $22.5 \pm 1.5$ & $21.9(19.0-25.3)$ \\
\hline Right/left side (n) & $17 / 25$ & - \\
\hline Tumor diameter $(\mathrm{cm})$ & $3.2 \pm 0.4$ & $3.2(1.8-3.9)$ \\
\hline \multicolumn{3}{|l|}{ Tumor location (n) } \\
\hline Polar & 25 & - \\
\hline Anterior & 7 & - \\
\hline Posterior & 9 & - \\
\hline R.E.N.A.L score & $5.2 \pm 1.6$ & $5(3-9)$ \\
\hline \multicolumn{3}{|l|}{ Perioperative } \\
\hline Surgery time (min) & $103 \pm 12$ & $99(86-145)$ \\
\hline $\mathrm{EBL}(\mathrm{ml})$ & $263 \pm 136$ & $227(105-725)$ \\
\hline WIT (min) & $23 \pm 3.6$ & $22.5(18-35)$ \\
\hline Transfusion (n) & 3 & - \\
\hline \multicolumn{2}{|l|}{ Postoperative complication } & - \\
\hline $\begin{array}{l}\text { eGFR absolute change of the } \\
\text { affected side }\left(\mathrm{ml} / \mathrm{min} / 1.73 \mathrm{~m}^{2}\right)\end{array}$ & $24.9 \pm 4.4$ & $24(16-38)$ \\
\hline \multicolumn{3}{|l|}{ Histology (n) } \\
\hline Clear-cell RCC & 34 & \\
\hline Papillary RCC & 1 & - \\
\hline Angiomyolipoma & 7 & - \\
\hline Margins negative (n) & 42 & - \\
\hline
\end{tabular}

BMI, body mass index; EBL, estimated blood loss; WIT, warm ischemia time; eGFR, estimated glomerular filtration rate; RCC, renal cell carcinoma; SD, standard deviation.

(SPSS, Inc., Chicago, IL, USA). Data are presented as the mean \pm standard deviation, or as the median and range. Continuous variables were compared using the paired Wilcoxon test, while nominal variables were analyzed using Pearson's $\chi^{2}$ test. $\mathrm{P}<0.05$ was considered to indicate a statistically significant difference.

\section{Results}

Patient characteristics. In total, 42 patients successfully underwent a LPN for renal masses of $<4 \mathrm{~cm}$. Patient demographics and tumor characteristics are presented in Table I. The mean tumor diameter was $3.2 \pm 0.4 \mathrm{~cm}$, the mean operative time was $103 \pm 12 \mathrm{~min}$, the estimated blood loss was $263 \pm 136 \mathrm{ml}$ and the mean warm ischemia time was $23 \pm 3.6 \mathrm{~min}$. A total of three patients $(7.1 \%)$ required a blood transfusion (Clavien grade II); however, no patients developed a renal arteriovenous fistula or required embolotherapy following surgery. The mean absolute reduction in the estimated glomerular filtration rate (eGFR) at 3-6 months 

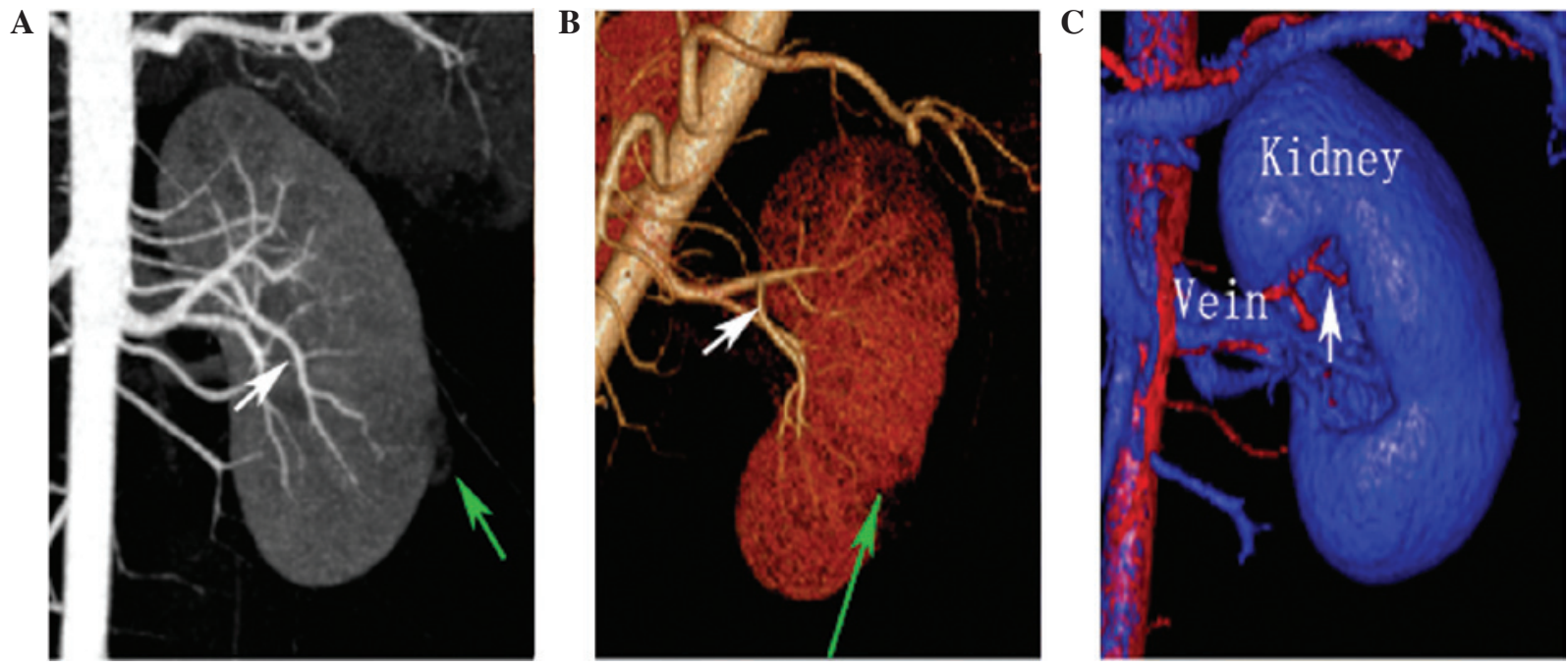

Figure 1. A single tumor-feeding artery identified using computed tomography angiography images. (A) Maximum intensity projection (MIP) image from the anterior view depicting the tumor-feeding branch (white arrow) and tumor (green arrow). (B) Tumor-feeding branch (white arrow) was annotated on the merged volume rendering (VR) and MIP image from the anterior view. (C) VR image from the anterior view displaying the tumor-feeding branch (white arrow) leading into the renal hilum.

A

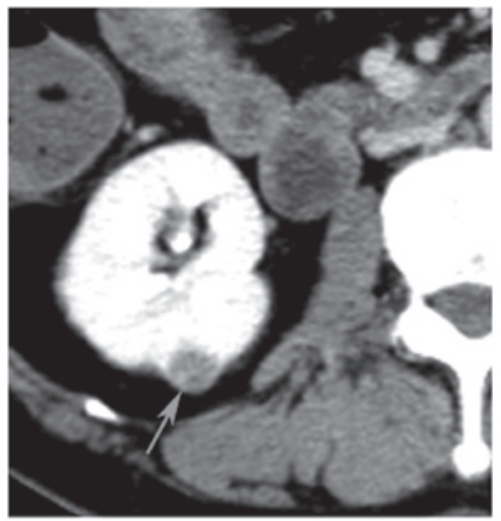

C

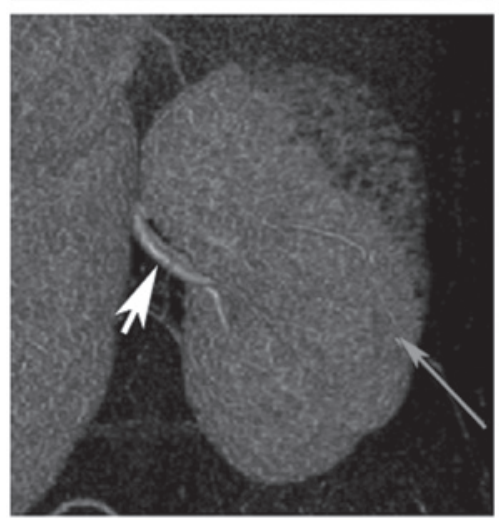

B

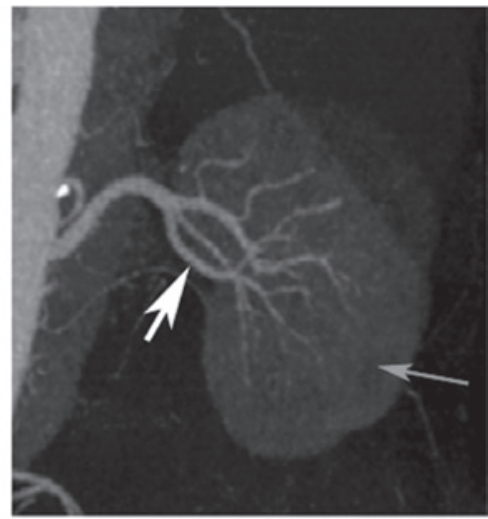

D

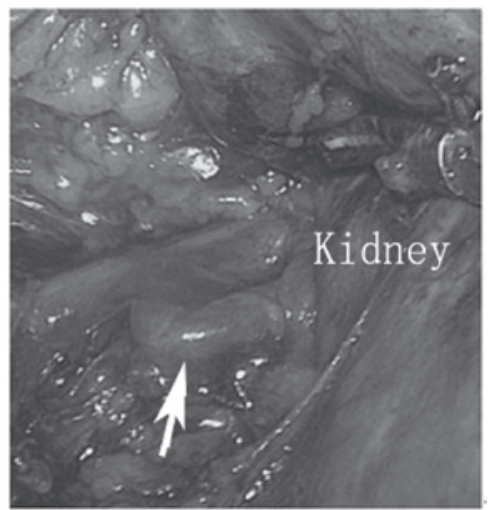

Figure 2. A 60-year-old woman underwent a right laparoscopic partial nephrectomy (LPN). (A) Axial maximum intensity projection image shows the tumor (grey arrow). (B and C) Tumor-feeding arteries (white arrow) were identified using computed tomography angiography images. (D) Target vessel (white arrow) was isolated during the LPN.

following surgery was $24.9 \pm 4.4 \mathrm{ml} / \mathrm{min} / 1.73 \mathrm{~m}^{2}$. Pathological examination detected clear-cell cancer in 34 cases, angiomyolipoma in seven cases and papillary cancer in one case, without positive margins.

Comparison between CTA and LPN surgical observations for renal arteries. CTA was performed in 42 patients, providing a preoperative map of the renal arteries.
Postprocessing images of all 42 cases accurately displayed the main renal trunks and vessel branches (Figs. 1 and 2). Comparison between the CTA and LPN observations for the renal arteries are summarized in Table II. Preoperatively, 57 renal arteries were identified using the CTA images. Among the 42 cases, CTA successfully detected 36 single renal arteries (97.3\%). Furthermore, a total of 5/6 accessory arteries were observed preoperatively using the CTA images 
Table II. Comparison between preoperative CTA predictions and LPN surgical observations for the renal arteries.

\begin{tabular}{lcccc}
\hline & \multicolumn{2}{c}{ LPN observations } & & \\
\cline { 2 - 3 } CTA prediction & 1 artery & 2 artery & Accuracy $(\%)$ & Overall accuracy $(\%)$ \\
\hline CTA results & - & - & 97.3 & 97.6 \\
1 artery & 36 & 1 & 100 & \\
2 artery & 0 & 5 & & \\
\hline
\end{tabular}

LPN, laparoscopic partial nephrectomy; CTA, computed tomography angiography.

Table III. Comparison between preoperative CTA predictions and LPN surgical observations for tumor-feeding arteries.

\begin{tabular}{|c|c|c|c|c|c|c|}
\hline \multirow[b]{2}{*}{ CTA prediction } & \multicolumn{3}{|c|}{ LPN observation } & \multirow[b]{2}{*}{ Accuracy $(\%)$} & \multirow[b]{2}{*}{ Overall accuracy (\%) } & \multirow[b]{2}{*}{ P-value } \\
\hline & 1 artery & 2 artery & 3 artery & & & \\
\hline 1 artery & 28 & 4 & 0 & 87.5 & & \\
\hline 2 artery & 1 & 7 & 1 & 77.8 & 85.7 & $0.839^{\mathrm{a}}$ \\
\hline 3 artery & 0 & 0 & 1 & 100 & & \\
\hline
\end{tabular}

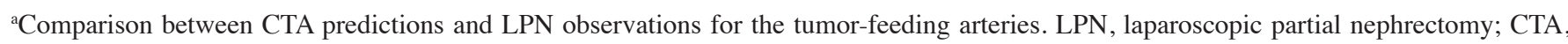
computed tomography angiography.

Table IV. Comparison between CTA predictions and LPN observations for the extrarenal length of the tumor-feeding arteries.

\begin{tabular}{lccc}
\hline Variable & CTA prediction & LPN observation & P-value \\
\hline $\begin{array}{l}\text { Extrarenal length of the tumor } \\
\text { feeding arteries (mm) }\end{array}$ & $30.2 \pm 2.8$ & $30.4 \pm 2.7$ & 0.183 \\
\hline
\end{tabular}

Results are expressed as the mean \pm standard deviation. LPN, laparoscopic partial nephrectromy; CTA, computed tomography angiography.
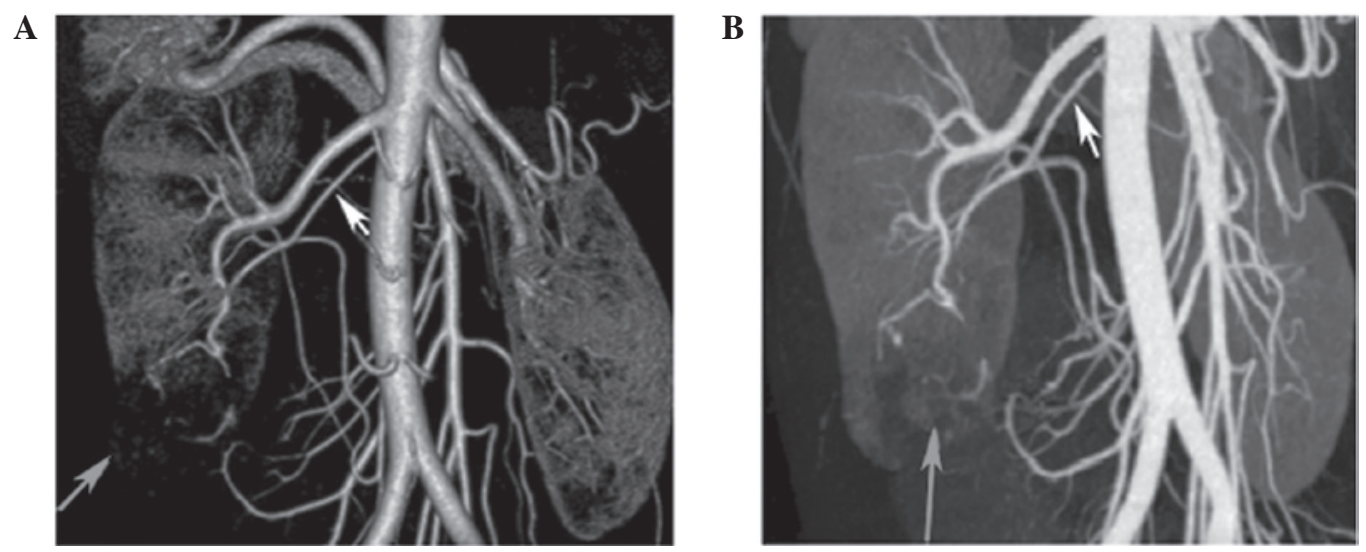

Figure 3. Accessory renal arteries were visualized using computed tomography angiography imaging for a 40-year-old woman that underwent a left laparoscopic partial nephrectomy. (A) Merged volume rendering and maximum intensity projection (MIP) image and (B) MIP image alone from the posterior view, depicting the accessory renal artery (white arrow) and the tumor (grey arrow).

(Fig. 3), while one accessory artery of $<2 \mathrm{~mm}$ in diameter was not detected. Thus, the accuracy of detection for the renal arteries using preoperative CTA was 97.6\% (41/42 arteries detected).
Comparison between CTA and LPN surgical observations for tumor-feeding arteries. Comparisons between preoperative CTA predictions and LPN surgical observations for the number of tumor-feeding arteries are summarized in Table III. 
The accuracy of CTA for the detection of renal tumor-feeding branches was $85.7 \%$. Statistical analysis identified no statistically significant differences between the CTA predictions and LPN observations with regard to the number of tumor-feeding arteries $(\mathrm{P}=0.839)$. The comparisons between the preoperative CTA and LPN surgical results for the extrarenal length of the tumor-feeding arteries are summarized in Table IV $(\mathrm{P}=0.183)$.

\section{Discussion}

A partial nephrectomy results in comparable oncological outcomes to a radical nephrectomy for small renal tumors, and is associated with a reduced risk of chronic kidney disease (1). As surgical techniques have improved, LPN has replicated the techniques of open partial nephrectomy for small renal tumors (10). Temporary hilar occlusion is commonly employed during a LPN; however, hilar occlusion may result in warm ischemic injury, which frequently impairs renal function (11). The safe upper limit for a period of warm ischemia remains controversial, ranging between 20 and $40 \mathrm{~min}(5,12)$. Thompson et al suggested that the warm ischemic period should be restricted as much as possible during a partial nephrectomy (13). In the present study, the warm ischemic period was $23 \pm 3.6 \mathrm{~min}$.

Hilar occlusion involves clamping the main renal vasculature to allow for improved visualization; however, the process may result in global renal ischemia. A number of techniques have been developed with the aim to minimize warm ischemic injury $(2,6,7)$. One approach, namely segmental renal artery clamping, eliminates global renal ischemia and minimizes the loss of renal function (4). Segmental renal artery clamping involves the selective clamping of renal arterial branches in order to eliminate global renal ischemia. In the present study, between May 2008 and December 2013, patients underwent a LPN with superselective clamping of the renal arterial branches. The mean absolute reduction in the eGFR at 3-6 months following surgery was $24.9 \pm 4.4 \mathrm{ml} / \mathrm{min} / 1.73 \mathrm{~m}^{2}$.

As the field of view during a LPN is limited, it is crucial that surgeons obtain preoperative knowledge of the renal arterial anatomy prior to performing selective clamping of the renal arterial branches. Although digital subtraction angiography is currently regarded as the gold standard for inspecting renal vascular anatomy, CTA is becoming increasingly used to evaluate renal arterial anatomy since the technique is less invasive, more accurate and more widely available (14). A previous study indicated that the sensitivity of CTA for preoperatively identifying renal arteries was $98.5 \%$ (15). Furthermore, Pozniak et al reported that the sensitivity of CTA was 100 and $93 \%$ for the detection of accessory arteries and prehilar arterial branches, respectively (16). In the present study, CTA correctly identified 36/37 single renal arteries, resulting in a $97.3 \%$ rate of accuracy.

In the majority of individuals, the kidney is supplied with blood by a single renal artery, and the prevalence of multiple renal arteries reportedly varies between 9 and $76 \%(17,18)$. The location of accessory arteries is crucial knowledge for surgeons to obtain prior to surgery, as the ligation of an accessory renal artery may lead to ischemic injury to the portion of the kidney supplied by that artery. However, hemorrhage may occur if a tumor-feeding accessory renal artery is not clamped, which may further affect the visualization of the laparoscopic field. Among the 42 cases investigated in the present study, 5/6 accessory arteries were preoperatively detected using CTA images, with only one accessory artery (diameter, $<2 \mathrm{~mm}$ ) undetected (Fig. 3). Therefore, preoperative CTA may aid surgeons in anticipating potential vascular variants and facilitating LNP.

The preoperative identification of tumor-feeding arteries may crucially affect the outcome of a LPN. Without effective guidance, renal arteries may go undetected or may be excessively clamped during the LPN, affecting visualization during surgery or resulting in a loss of renal function. The renal artery is typically divided into segmental arteries at the renal hilum. With reformatting techniques, CTA can be used to accurately elucidate the number, branching pattern and location of the renal arteries. Thus, surgeons may preoperatively identify tumor-feeding arteries using CTA images. In the present study, the accuracy of the identification of tumor-feeding arteries via 256-channel CTA was evaluated by comparing the CTA prediction with the actual segmental arteries clamped during surgery. The accuracy of CTA in detecting the renal tumor-feeding branches was $85.7 \%$, and statistical analysis indicated no statistically significant difference between the CTA predictions and LPN observations with regard to the number of tumor-feeding arteries ( $\mathrm{P}=0.839$; Fig. 2).

The extrarenal length of the tumor-feeding arteries is a significant factor for the selective clamping of renal arterial branches. Previously, Nohara et al reported that tumor-feeding arteries of $>10 \mathrm{~mm}$ in extrarenal length were necessary for LPN to be performed (19). Furthermore, a previous study by Weld et al (20) indicated that the required extrarenal length of tumor-feeding arteries for conducting a LPN was $31 \mathrm{~mm}$. In addition, Kang et al reported that tumors in the lower pole may be more amenable to segmental artery clamping due to the presence of longer tumor-feeding arteries (8). In the present study, the extrarenal length of the tumor-feeding arteries was preoperatively evaluated using CTA, and statistical analysis indicated no statistically significant difference between the CTA prediction and LPN observation of the extrarenal length of the tumor-feeding arteries $(\mathrm{P}=0.183)$. In total, 42 patients successfully underwent a LPN with superselective clamping of the renal arterial branches, and no patients were converted to a LPN with hilar occlusion. Therefore, the use of CTA may allow surgeons to preoperatively evaluate the feasibility of segmental artery clamping, and thus avoid unnecessary dissection of the segmental artery.

A number of limitations were identified in the present study. Firstly, the number of enrolled patients was relatively limited; thus, further studies with larger population samples are required to verify the present results. Secondly, there may be a selection bias due to the retrospective nature of the study.

In conclusion, CTA may be used to preoperatively produce a map of the renal arteries, subsequently providing a surgical advantage by optimizing the detection of tumor-feeding arteries. Therefore, the use of CTA may facilitate successful segmental renal artery clamping during a LPN.

\section{Acknowledgements}

The authors thank the medical staff from the Department of Urology, Dongguan People's Hospital (Dongguan, China) 
for supporting the study, and Professors Xiaolin Zheng and Tao Hu of the Department of Radiology, Dongguan People's Hospital for providing CT materials. In addition, the authors thank the patients for volunteering to participate in the study. The study was supported by the Science and Technological Program (no. 2012105102027) for Dongguan's Higher Education, Science and Research and Health Care Institutions.

\section{References}

1. Van Poppel H, Da Pozzo L, Albrecht W, et al: A prospective, randomised EORTC intergroup phase 3 study comparing the oncologic outcome of elective nephron-sparing surgery and radical nephrectomy for low-stage renal cell carcinoma. Eur Urol 59: 543-552, 2011.

2. Gill IS, Eisenberg MS, Aron M, Berger A, Ukimura O, et al: 'Zero ischemia' partial nephrectomy: Novel laparoscopic and robotic technique. Eur Urol 59: 128-134, 2011.

3. Gill IS, Kavoussi LR, Lane BR, et al: Comparison of 1,800 laparoscopic and open partial nephrectomies for single renal tumors. J Urol 178: 41-46, 2007.

4. Shao P, Qin C, Yin C, Meng X, Ju X, Li J, Lv Q, Zhang W and $\mathrm{Xu} Z$ : Laparoscopic partial nephrectomy with segmental renal artery clamping: Technique and clinical outcomes. Eur Urol 59: 849-855, 2011.

5. Choi JD, Park JW, Choi JY, Kim HS, Jeong BC, Jeon SS, Lee HM, Choi HY and Seo SI: Renal damage caused by warm ischaemia during laparoscopic and robot-assisted partial nephrectomy: An assessment using Tc 99m-DTPA glomerular filtration rate. Eur Urol 58: 900-905, 2010.

6. Peyronnet B, Baumert H, Mathieu R, et al: Early unclamping technique during robot-assisted laparoscopic partial nephrectomy can minimise warm ischaemia without increasing morbidity. BJU Int 114: 741-747, 2014.

7. Hsi RS, Macleod LC, Gore JL, Wright JL and Harper JD: Comparison of selective parenchymal clamping to hilar clamping during robotic-assisted laparoscopic partial nephrectomy. Urology 83: 339-344, 2014.

8. Kang WY, Sung DJ, Park BJ, et al: Perihilar branching patterns of renal artery and extrarenal length of arterial branches and tumour-feeding arteries on multidetector CT angiography. $\mathrm{Br}$ J Radiol 86: 20120387, 2013.
9. Rastogi N, Sahani DV, Blake MA, Ko DC and Mueller PR: Evaluation of living renal donors: Accuracy of three-dimensional 16-section CT. Radiology 240: 136-144, 2006.

10. Gill IS, Desai MM, Kaouk JH, et al: Laparoscopic partial nephrectomy for renal tumor: Duplicating open surgical techniques. J Urol 167: 469-7, discussion 475-476, 2002.

11. Porpiglia F, Fiori C, Bertolo R, Morra I, Russo R, Piccoli G, Angusti T and Podio V: Long-term functional evaluation of the treated kidney in a prospective series of patients who underwent laparoscopic partial nephrectomy for small renal tumors. Eur Urol 62: 130-135, 2012.

12. Porpiglia F, Fiori C, Bertolo R, et al: The effects of warm ischaemia time on renal function after laparoscopic partial nephrectomy in patients with normal contralateral kidney. World J Urol 30: 257-263, 2012.

13. Thompson RH, Lane BR, Lohse CM, et al: Every minute counts when the renal hilum is clamped during partial nephrectomy. Eur Urol 58: 340-345, 2010.

14. Türkvatan A, Ozdemir M, Cumhur T and Olçer T: Multidetector CT angiography of renal vasculature: Normal anatomy and variants. Eur Radiol 19: 236-244, 2009.

15. Raman SS, Pojchamarnwiputh S, Muangsomboon K, Schulam PG, Gritsch HA and Lu DS: Utility of 16-MDCT angiography for comprehensive preoperative vascular evaluation of laparoscopic renal donors. AJR Am J Roentgenol 186: 1630-1638, 2006.

16. Pozniak MA, Balison DJ, Lee FT Jr, Tambeaux RH, Uehling DT and Moon TD: CT angiography of potential renal transplant donors. Radiographics 18: 565-587, 1998.

17. Uflacker R (ed): Abdominal aorta and branches. In: Atlas of Vascular Anatomy: An Angiographic Approach. 2nd edition. Lippincott Williams \& Wilkins, Philadelphia, PA, pp111-222, 2006.

18. Satyapal KS, Haffejee AA, Singh B, Ramsaroop L, Robbs JV and Kalideen JM: Additional renal arteries: Incidence and morphometry. Surg Radiol Anat 23: 33-38, 2001.

19. Nohara T, Fujita H, Yamamoto K, Kitagawa Y, Gabata T and Namiki M: Modified anatrophic partial nephrectomy with selective renal segmental artery clamping to preserve renal function: A preliminary report. Int J Urol 15: 961-966, 2008.

20. Weld KJ, Bhayani SB, Belani J, Ames CD, Hruby G and Landman J: Extrarenal vascular anatomy of kidney: Assessment of variations and their relevance to partial nephrectomy. Urology 66: 985-989, 2005. 